\title{
Wettability Enhancement of Polystyrene with Electron Cyclotron Resonance Plasma with Argon
}

\author{
S. Guruvenket, ${ }^{1}$ Manoj Komath, ${ }^{2}$ S. P. Vijayalakshmi, ${ }^{3}$ A. M. Raichur, ${ }^{3}$ G. Mohan Rao ${ }^{1}$ \\ ${ }^{1}$ Department of Instrumentation, Indian Institute of Science, Bangalore 560 012, India \\ ${ }^{2}$ Biomedical Technology Wing, Sree Chithra Tirunal Institute of Medical Sciences and Technology, \\ Thiruvananthapuram 695 012, India \\ ${ }^{3}$ Department of Metallurgy, Indian Institute of Science, Bangalore 560 102, India
}

Received 4 September 2002; accepted 21 January 2003

\begin{abstract}
Polystyrene cell-culture substrates were treated with argon glow discharge to make their surfaces hydrophilic. The process was novel in that it used a microwave electron cyclotron resonance (ECR) source for polymer surface modification. The substrates were processed at different microwave powers and time periods, and the surface modification was assessed with by measurement of the water contact angle. A decrease in contact angle was observed with increasing microwave power and processing time. Beyond a certain limit of power and duration of exposure, however, surface deterioration occurred. The optimum conditions for making the surfaces hydrophilic without deteri-
\end{abstract}

oration of the samples were identified. The plasma parameters were assessed by Langmuir probe measurement. Fourier transform infrared spectroscopy with attenuated total reflectance showed evidence for the induction of hydrophilicity on the surface. The surface micromorphology was examined with scanning electron microscopy. The results prove that the ECR glow discharge was an efficient method for enhancing the wettability of the polymer surfaces. (c) 2003 Wiley Periodicals, Inc. J Appl Polym Sci 90: 1618-1623, 2003

Key words: biocompatibility; cold plasma; crosslinking; hydrophilic polymers; polystyrene

\section{INTRODUCTION}

Enhancing the wettability of polymer surfaces is a subject of research interest because most common polymers are hydrophobic in nature, which makes them unsuitable for some important biomedical and industrial applications. ${ }^{1,2}$ Several surface-processing techniques have been devised so as to induce hydrophilicity on the surface of such polymers and to preserve desirable bulk properties. The techniques for wettability enhancement fall into three classes: wet chemical processing, plasma treatment (glow discharge, corona, and flame), and radiation treatment (ultraviolet radiation and laser treatment). The choice of technique depends on the final application of the polymer surface. $^{2}$

The need for polymer surface wettability becomes crucial in applications such as cell culture. ${ }^{3,4}$ Glass cell-culture wares, despite their excellent cell compatibility and optical transparency, are now being replaced by disposable plastics, mainly because of problems with sterility and contamination in reuse and the risk of breakage. Polystyrene is the preferred candidate for cell-culture wares because of its good optical qualities and handling characteristics and because it

Correspondence to: G. Mohan Rao (gmrao@isu.iisc.ernet.in).

Journal of Applied Polymer Science, Vol. 90, 1618-1623 (2003) (C) 2003 Wiley Periodicals, Inc. provides a nontoxic surface for cell growth, which gives reproducible results. However, surface modification is required because the material is basically hydrophobic. $^{5}$

Glow discharge plasmas are generally used for surface modification for biomedical applications because the processes involved are solvent-free and dry, the consumption of chemicals is extremely low, and the need for sterilization of the product is eliminated. Moreover, the processes are controllable, the surfaces can be treated homogeneously, and the surface chemistry can be tailored for its end use. ${ }^{1,2}$ Glow discharge plasmas are created by the induction of electric potential (direct current) or electromagnetic radiation (radio-frequency radiation or microwaves) to a low-pressure gas. The ionized gas (i.e., the discharge) contains energetic species, ions and electrons, which act on the surface under treatment to change its physicochemical properties. ${ }^{1,2}$

In most of the existing studies on polymer surface modification with plasmas, plasma parameters such as ion density and ion energy were not determined, and these are crucial factors in the surface modification of the polymer. Hence, it is important to correlate the plasma parameters with the features of the plasma-modified polymer surface. This study demonstrated the use of the electron cyclotron resonance (ECR) technique to create a glow discharge plasma for polymer surface processing. High ion density in the 
discharge and the moderate energy of the species made it a useful tool for the purpose. Polystyrene tissue-culture substrate surfaces were treated in argon plasma created in an ECR reactor to enhance wettability with an aim to make the surface more cellcompatible. Variations in the plasma parameters were tracked with a Langmuir probe. Variations in wettability were determined with water contact angle measurements, and the surface structural changes were investigated with Fourier transform infrared spectroscopy with attenuated total reflectance (FTIR-ATR). Scanning electron microscopy (SEM) was used for surface micromorphology observations.

\section{EXPERIMENTAL}

\section{Equipment}

The ECR technique basically uses microwave power for the excitation of the glow discharge, and the electron motion is geared by an axial, static magnetic field. The strength of the magnetic field is adjusted in such a way that the circular motion of the electron in the discharge is in resonance with the microwave frequency. The magnetic field required for resonance $(B)$ is given by the equation $B=m \omega / q$, where $\omega$ is the frequency of the microwave and $m$ and $q$ are the mass and the charge of the electron, respectively. The result is a substantial increase in ion density, two to three orders higher than with conventional techniques.

The schematic diagram of an ECR reactor is shown in Figure 1. It contains a source chamber with two electromagnet coils wound around and a processing chamber underneath, both connected to a vacuum system. Microwave power, generated in a continuouswave magnetron source $(2.45 \mathrm{GHz})$, is fed from the top through a quartz vacuum window with the help of appropriate waveguides. The electromagnets are positioned to get a "magnetic mirror" inside the source chamber. The gas (argon) is admitted to the source chamber with a flow controller, and the glow discharge is generated by the application of microwave power. The downstream plasma, extending into the processing chamber, is used for surface modification.

The ECR source has significant advantages over other techniques, such as radio-frequency glow discharge, that are used in polymer surface processing. It can be operated at low pressures, in the range of $10^{-4}$ to $10^{-3}$ mbar, which minimizes the chance for gasphase contamination in processing. The ion densities are higher (of the order of $10^{11}$ to $10^{13} / \mathrm{cm}^{3}$ ), and the energy of the species or ions in the glow discharge is moderate $(<50 \mathrm{eV})$. These advantages enable the faster processing of the surface without the alteration of the polymer structure in the bulk. Moreover, the parameters (energy and density of the ions/species bombarding the sample material) can be controlled

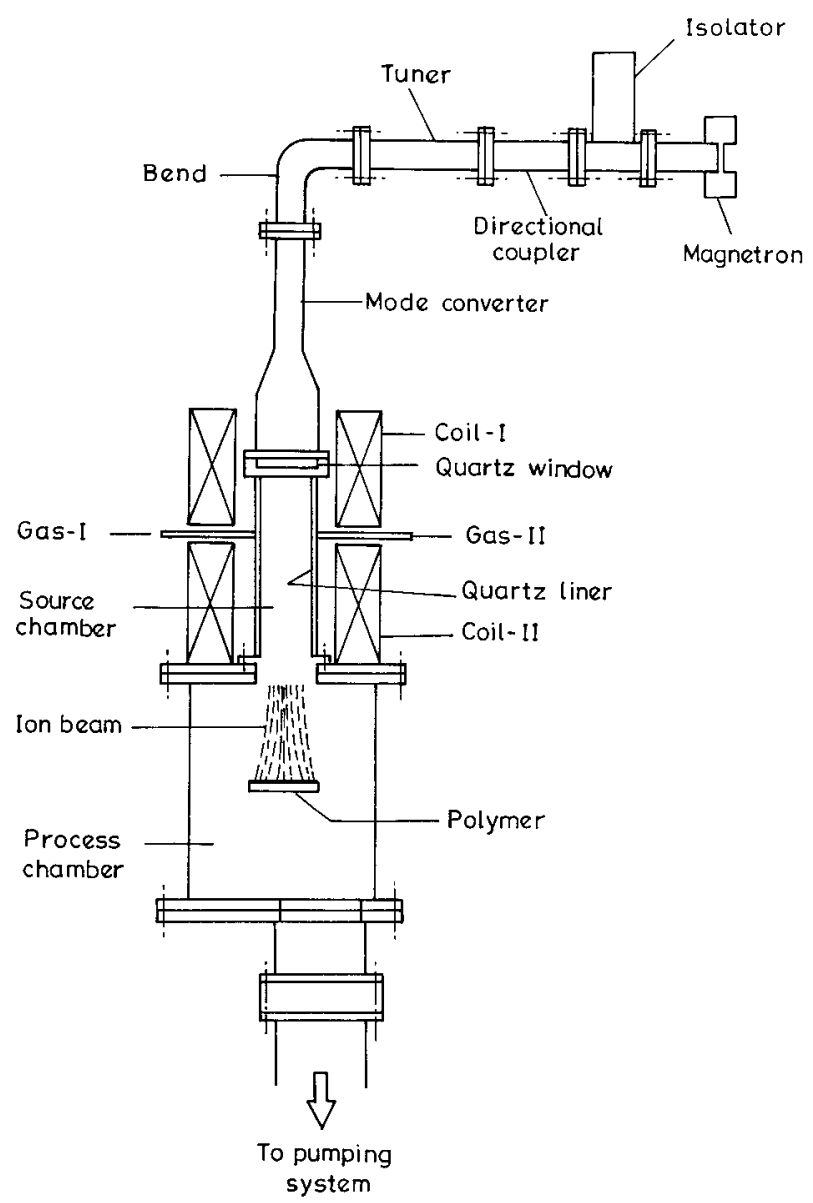

Figure 1 Schematic diagram of an ECR reactor.

conveniently by the manipulation of the strength and configuration of the magnetic field in the source chamber and by the adjustment of the microwave power. ${ }^{6}$

\section{Procedure}

An ECR plasma source constructed in-house, the details of which were reported earlier, ${ }^{7}$ was used for the experiment. The chambers were evacuated to a base pressure of $10^{-6} \mathrm{mbar}$, and the working pressure was maintained at $10^{-3}$ mbar by the admission of argon gas. The sample was kept $10 \mathrm{~cm}$ from the exit of the source chamber. The currents in the upper and lower magnetic coils were maintained at 60 Amps. We carried out the surface treatment systematically by changing the microwave power and the processing time.

Ultrasonically cleaned pieces of polystyrene tissueculture petri dishes with dimensions of $1 \times 1 \mathrm{~cm}$ were used as samples. Preliminary investigations showed that microwave powers up to $100 \mathrm{~W}$ were not sufficient to create a substantial increase in the wettability and that power values higher than $300 \mathrm{~W}$ caused deterioration of the polymer surface. Hence, by varying the processing time and the microwave power, we 


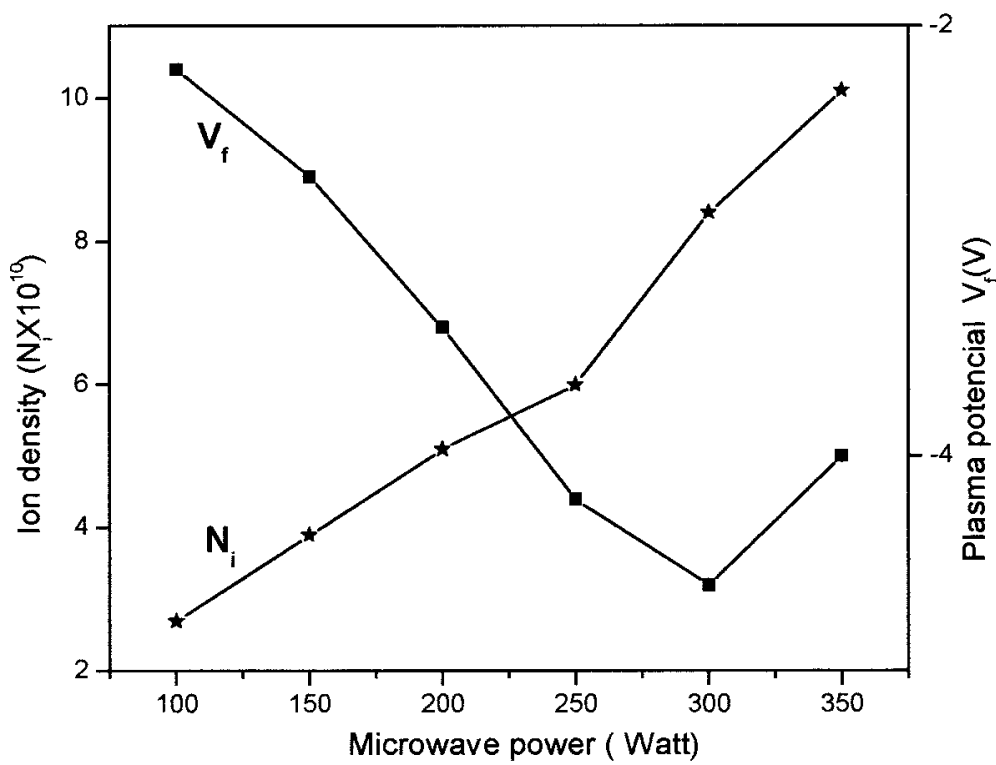

Figure 2 Changes in the ion density $\left(N_{i}\right)$ and plasma potential $\left(V_{f}\right)$ of the ECR plasma.

determined the optimum values at a fixed distance of the sample and pressure.

\section{Characterization}

Plasma analysis

Plasma parameters such as ion density and electron temperature play a vital role in the surface modification of the polymer. Hence, it is necessary to determine the plasma parameters to have a better understanding of the basic reactions occurring on the surface of the polymer. In this investigation, a Langmuir probe was used to measure the plasma parameters. The Langmuir probe basically consisted of a cylindri- cal probe (tungsten wire $0.25 \mathrm{~mm}$ in diameter and 5 $\mathrm{mm}$ in length) placed perpendicular to the plasma stream. A potential was imposed on the metal probe relative to the ground, which was in contact with plasma. The plasma parameters could be extracted from the shape of the current-voltage characteristics. ${ }^{8}$

Polymer surface analysis

Surface modifications can easily be characterized by the measurement of the contact angles of the surface with liquids. ${ }^{9}$ In this study, the sessile drop technique was used, with double-distilled, deionized water drops. Glass and Teflon were used as standards for the

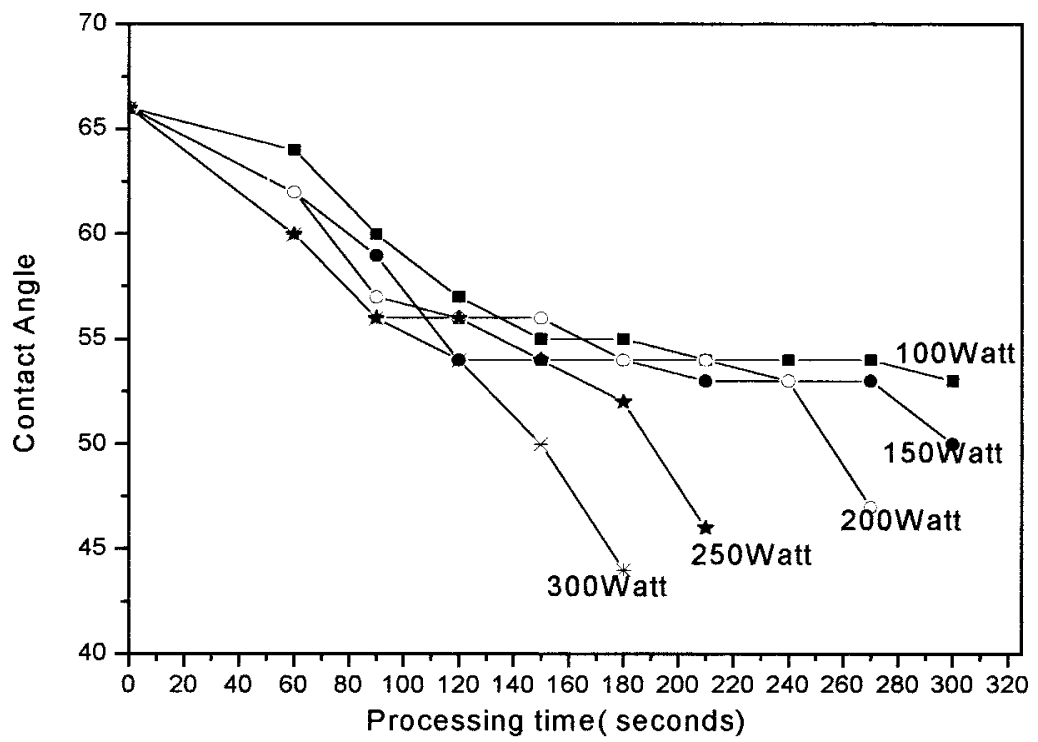

Figure 3 Contact angle as a function of microwave power and processing time. 
TABLE I

Contact Angle $\left(^{\circ}\right)$ as a Function of Microwave Power and Processing Time

\begin{tabular}{ccccccccccc}
\hline \multirow{2}{*}{$\begin{array}{l}\text { Microwave } \\
\text { power (W) }\end{array}$} & 0 & 60 & 90 & 120 & 150 & 180 & 210 & 240 & 270 & 300 \\
\cline { 2 - 26 } & 66 & 64 & 60 & 57 & 55 & 55 & 54 & 54 & 54 & 53 \\
100 & 66 & 62 & 59 & 54 & 54 & 54 & 53 & 53 & 53 & 50 \\
200 & 66 & 62 & 57 & 56 & 56 & 54 & 54 & 53 & 47 & - \\
250 & 66 & 60 & 56 & 56 & 54 & 52 & 46 & - & - & - \\
300 & 66 & 60 & 56 & 54 & 50 & 44 & - & - & - & - \\
\hline
\end{tabular}

hydrophilic and hydrophobic surfaces, respectively. The measurements were taken in comparison with the untreated surface.

The surface modification in a glow discharge is confined only to some nanometers below the surface. FTIR-ATR is capable of bringing out surface information. ${ }^{10}$ This well-known technique for the analysis of polymer surfaces was used in this study. The spectra of the treated samples and the untreated sample were compared to observe the changes.

The surfaces, immediately after treatment, were coated with gold, and their micromorphologies were observed under a scanning electron microscope. The changes in the surface morphologies of the polymers exposed to different levels of microwave power for different times were observed.

\section{RESULTS AND DISCUSSION}

\section{Langmuir probe analysis}

The probe bias was varied from -60 to $+60 \mathrm{~V}$, and the probe current was measured. A plot between the probe voltage and the probe current was made (current-voltage characteristic). Ion density and plasma potential were determined from the curve with standard methods on the assumption that ions and electrons in the ECR plasma followed Maxwellian distribution. Figure 2 shows the variation of the plasma potential and the ion density as a function of microwave power.

As clearly shown in Figure 2, as the microwave power increased, the ion density increased, and at the same time, the plasma potential decreased. The change in the plasma potential indicated the change in the ion energy. Beyond $300 \mathrm{~W}$ of microwave power, the plasma potential again started increasing. This trend of the plasma potential showed that when the microwave power exceeded $300 \mathrm{~W}$, there was drastic change in the ion density and the ion energy. The ion energy is an important factor; it induces chemical reactions and also causes surface heating effects, which in turn, deteriorate the polymer surface. Also, a higher ion energy increases the roughness of the polymer surface, which in turn, affects the contact angle

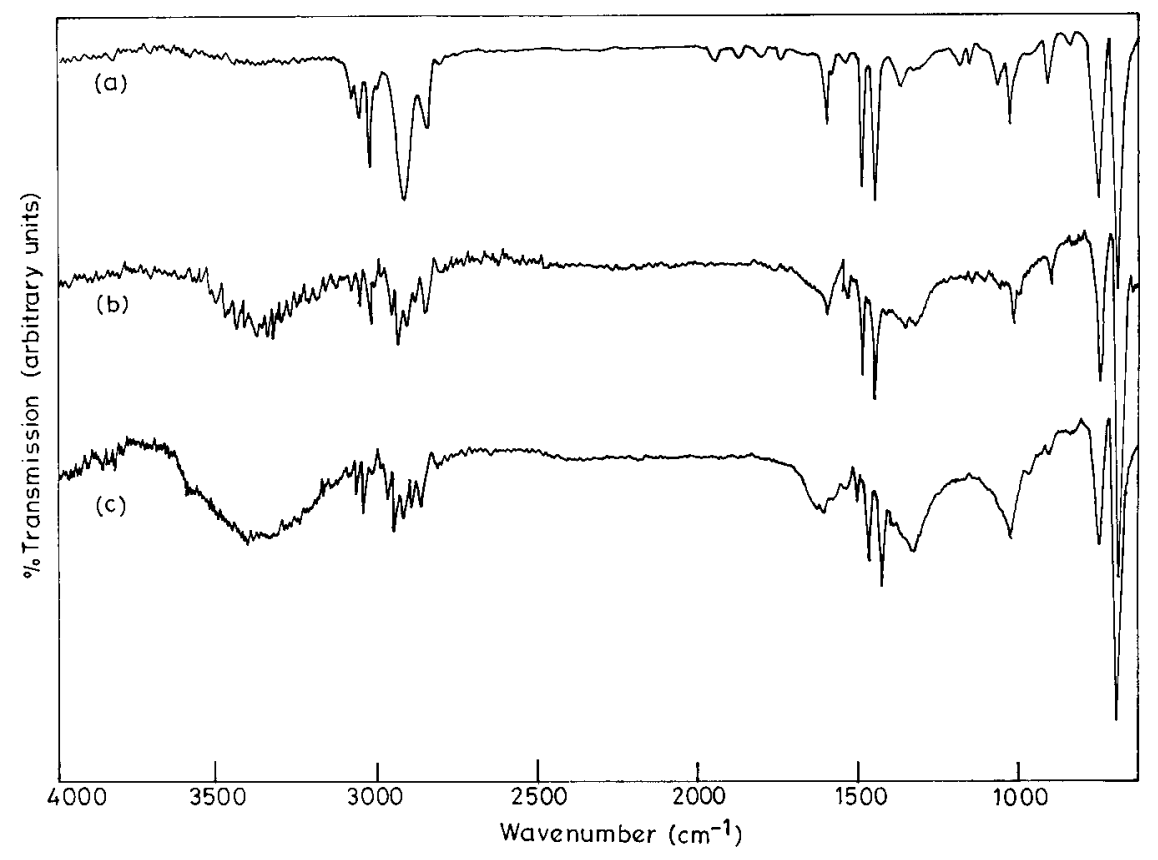

Figure 4 FTIR-ATR spectra of treated and untreated polystyrene: (a) untreated polystyrene and polystyrene treated at (b) $100 \mathrm{~W}$ for $240 \mathrm{~s}$ and (c) $300 \mathrm{~W}$ for $120 \mathrm{~s}$. 
measurement. ${ }^{11-13}$ However, a higher ion density and lower ion energy favors surface activation rather than increasing the surface roughness or affecting the bulk properties of the polymer.

\section{Contact angle measurements}

In static contact angle determination with doubly deionized water, polytetrafluoroethylene (PTFE) (the standard hydrophobic surface) and glass (the standard hydrophilic surface) were used to validate the measurements. For these materials, the measured contact angles were 110 and $14^{\circ}$, respectively, which agreed with reported values. ${ }^{9,14}$ The water contact angles of the polystyrene samples treated with different levels of microwave power for different processing times are shown in Figure 3 and also in Table I. The treated samples showed a decrease in the contact angle with increasing microwave power and processing time.

\section{FTIR-ATR analysis}

We performed the FTIR-ATR analysis by pressing the processed surfaces of the samples onto the ATR attachment. All of the samples treated at different microwave powers for different processing times showed similar FTIR-ATR spectra. The comparative FTIR-ATR spectra of the treated and untreated samples are shown in Figure 4. A comparison of the spectra shows broad bands around 3300 and $1600 \mathrm{~cm}^{-1}$, which corresponded to the hydroxyl group $\left(\mathrm{OH}^{-}\right) .^{15}$ This, possibly, occurred because of the absorption of the water vapor present in the atmosphere on the modified surface. This was an indication of the increase in hydrophilicity because of argon glow discharge processing. The contact angle measurements supported this observation. There was no detectable change in the characteristic peaks of styrene.

\section{SEM studies}

The surface morphologies of samples are shown in Figure $5(\mathrm{a}-\mathrm{c})$. The images of the treated samples in comparison with the untreated sample indicated that surface morphology changes occurred during the glow discharge treatment. The surface changes were minimal in the sample that was treated at $100 \mathrm{~W}$ for the shorter period of time, whereas the samples that were treated with the higher power and exposed for the longer period showed deterioration of the polymer surface. At the intermediate value of the power and the exposure time, a very moderate change in the surface morphology of the polymer was induced.

A comparison of the contact angle values of the polystyrene samples treated in argon plasma with the plasma parameters such as ion energy and ion density

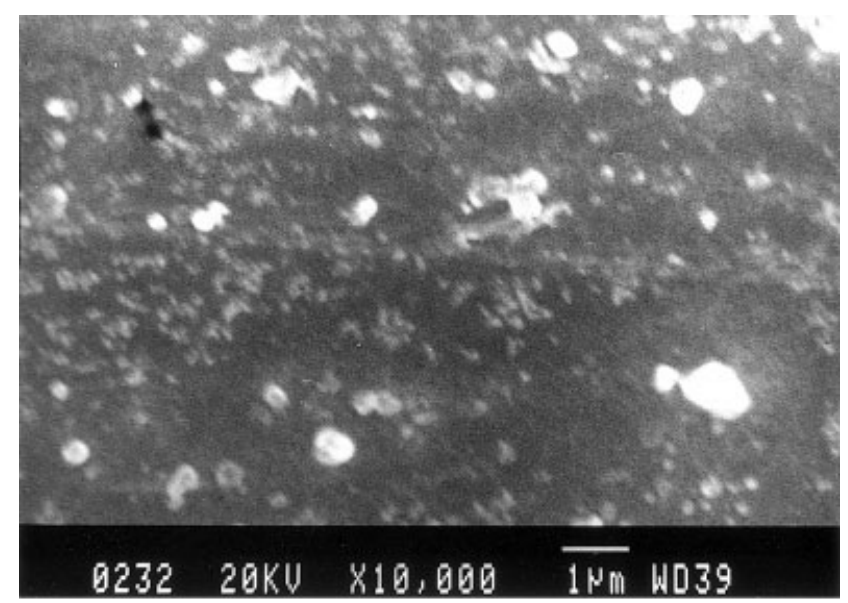

(a)

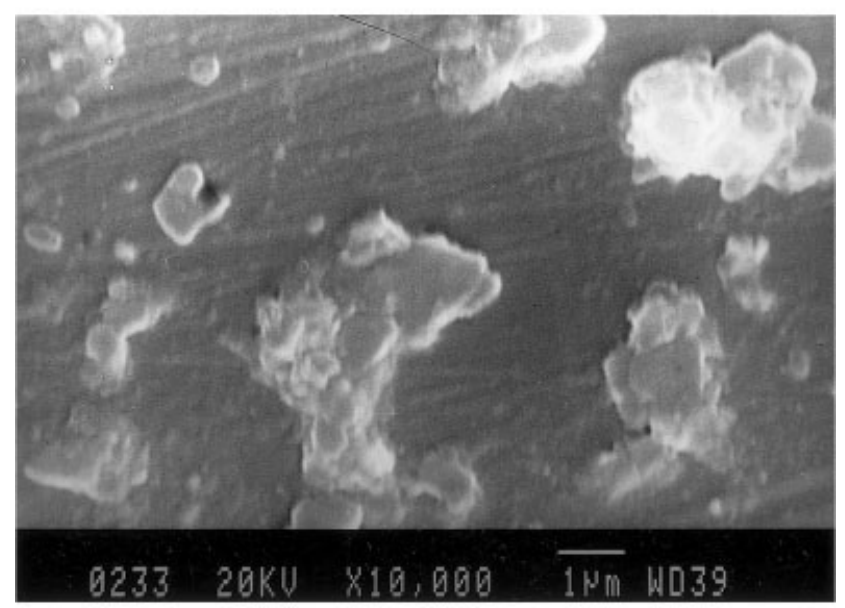

(b)

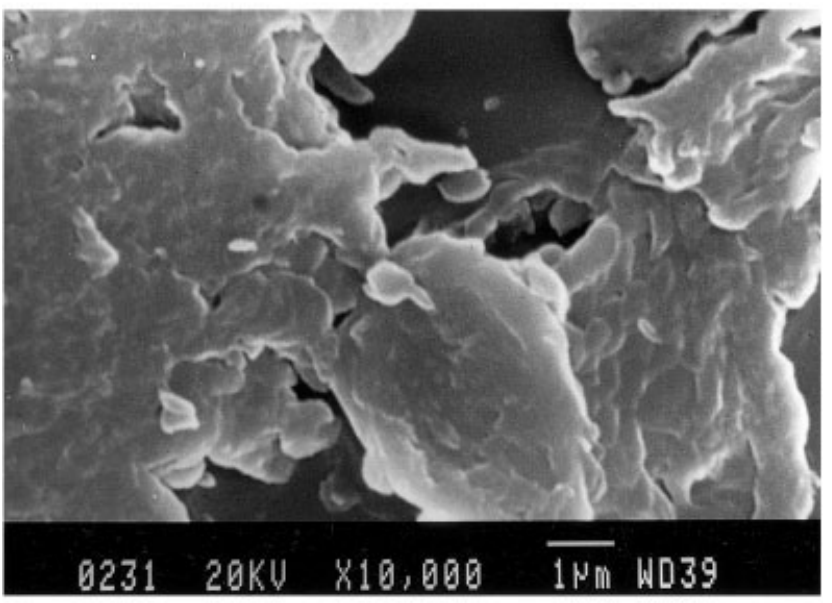

(c)

Figure 5 Electron micrographs of the polystyrene surfaces: (a) untreated polystyrene and polystyrene treated at (b) 100 and (c) $300 \mathrm{~W}$ for $240 \mathrm{~s}$.

clearly indicated that the ion energy and the ion flux/ density played a crucial role in the surface modification of the polystyrene sample. With increasing micro- 
wave power, the ion density increased, and the plasma potential decreased; this trend was observed up to $300 \mathrm{~W}$, and beyond $300 \mathrm{~W}$, the plasma potential started increasing. This means that at more than 300 $\mathrm{W}$, more energetic ions came and bombarded the surface. The contact angle value decreased with increasing microwave power and also with the treatment time at a constant power. When the microwave power was above $300 \mathrm{~W}$, the surface deteriorated. A similar effect was observed when the treatment time was increased beyond a certain value, even at the lower microwave power. This clearly showed that the plasma parameters had a definite effect on the surface of the polymer. The surface modification and changes in the wettability state were also indicated by the FTIR-ATR spectra, which showed strong $(-\mathrm{OH})$ absorption groups on the surface of the polymer. This was clear evidence of the changes in the surface nature, whereas the polymer that was treated for a longer period or at higher power was damaged. The SEM images clearly showed the surface damage of the polymer.

At higher microwave powers and longer treatment times, damage to the surface of the polymer occurred [which is evident in the SEM micrograph in Fig. 5(c)]. The contact angle value measured on the polymer surface not only depends on the functional group that is present on the surface but also on physical parameters such as surface roughness. In our case, it was evident that the surface roughness increased and played a crucial role at higher plasma powers and longer treatment times. Hence, the change in the contact angle could also be attributed to the roughness change at the higher powers and treatment times.

These results indicate that the ECR glow discharge with argon increased the surface wettability of the polystyrene surface. The description of the exact mechanism behind this wettability enhancement is difficult because the processes occurring at the polymer surface during glow discharge treatment were highly complex. Generally, a glow discharge creates a wettability change on the polymer surface through oxidation, unsaturation effects, electrostatic charging, and surface morphology changes. ${ }^{1,2}$ In the case of argon glow discharge over polystyrene, material degradation (hydrogen abstraction from styrene and chain scission) was most likely. A plausible explanation of this case of wettability enhancement, in addition to the molecular weight changes, may be the minor oxidation of the treated surface when it was exposed to air after treatment. ${ }^{16}$ Further studies are in progress to identify the exact reasons for the behavior of the plasma treated surface with X-ray photoelectron spectroscopy.

\section{CONCLUSIONS}

Polystyrene surfaces were treated in an argon glow discharge created through the ECR technique. Langmuir probe analysis showed variation in the ion density and plasma potential with microwave power. The results of the water contact angle measurements and FTIR-ATR studies on the plasma-treated surfaces showed an enhancement in the wettability. A correlation between the plasma parameter and the contact angle was made. The optimization of the plasma parameters for polystyrene was performed.

The increase in the hydrophilicity of the polystyrene surface on processing in the ECR argon glow discharge showed that the ECR source was an effective tool for the surface processing of the polymer.

The authors thank T. V. Kumary, Sree Chithra Tirunal Institute of Medical Sciences and Technology, Thiruvananthapuram, India, for information on cell culture.

\section{References}

1. Sheu, M. S.; Hudson, D. M.; Loh, I. H. In The Encyclopedic Handbook of Biomaterials and Bio-engineering: Part A; Marcel Dekker: New York, 1995; Vol. 1, p 865.

2. Chan, C. M.; Ko, T. M. Surf Sci Rep 1996, $24,1$.

3. Freshney, R. I. In Culture of Animal Cells, 3rd ed.; Wiley-Liss: New York, 1994; 71.

4. Dewez, L.; Doren, A.; Schneider, Y. J.; Rouxhet, P. G. Biomaterials 1999, 20, 547.

5. Onyiriuka, E. C. J Appl Polym Sci 1993, 47, 2187.

6. Asmussen, J. J Vac Sci Technol A 1989, 7, 883.

7. Deenama Varghese, K.; Mohan Rao, G. Rev Sci Instrum 2000, 71, 467.

8. Chapman, B. Glow Discharge Process; Wiley: New York, 1980.

9. Adamson, A. W. In Physical Chemistry of Surfaces, 3rd ed.; Wiley,: New York, 1976.

10. Rochotzki, R.; Nitschke, M.; Artz, M.; Meichsner, J. Phys Status Solidi A 1994, 145, 289.

11. Tzeng, S.; Chen, H. J.; Wang, Y. Y.; Wab, C. C. Surf Coat Technol 1997, 89, 108.

12. Dwight, D. W.; Riggs, W. M. J Colloid Interface Sci 1974, 47, 650.

13. Garbassi, F.; Morra, M.; Occhiello, E. In Polymer Surfaces: From Physics to Technology; Wiley: New York, 1994; Chapter 4.

14. Dann, R. J Colloid Interface Sci 1970, 32, 302.

15. Silverstein, R. M.; Bassler, G. C.; Terence. In Spectrometric Identification of Organic Compounds, 5th ed.; Wiley: New York, 1991; Chapter 3.

16. Yasuda, H. J Macromol Sci Chem 1976, 10, 383. 